\title{
Sensorimotor Cortical Oscillations during Movement Preparation in 16p11.2 Deletion Carriers
}

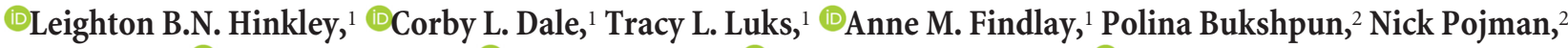

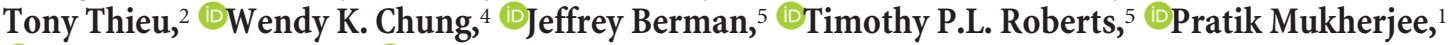 \\ Elliott H. Sherr, ${ }^{2,3 *}$ and ${ }^{-S}$ Srikantan S. Nagarajan ${ }^{1 *}$ \\ Departments of ${ }^{1}$ Radiology and Biomedical Imaging, ${ }^{2}$ Neurology, and ${ }^{3}$ Pediatrics, University of California, San Francisco, San Francisco, California 94143, \\ ${ }^{4}$ Department of Medicine, Columbia University, New York, New York, 10032, and 5Department of Radiology, Children's Hospital of Philadelphia, \\ Philadelphia, Pennsylvania 19104
}

Sensorimotor deficits are prevalent in many neurodevelopmental disorders like autism, including one of its common genetic etiologies, a $600 \mathrm{~kb}$ reciprocal deletion/duplication at 16p11.2. We have previously shown that copy number variations of $16 \mathrm{p} 11.2$ impact regional brain volume, white matter integrity, and early sensory responses in auditory cortex. Here, we test the hypothesis that abnormal cortical neurophysiology is present when genes in the 16p11.2 region are haploinsufficient, and in humans that this in turn may account for behavioral deficits specific to deletion carriers. We examine sensorimotor cortical network activity in males and females with 16p11.2 deletions compared with both typically developing individuals, and those with duplications of 16p11.2, using magnetoencephalographic imaging during preparation of overt speech or hand movements in tasks designed to be easy for all participants. In deletion carriers, modulation of beta oscillations $(12-30 \mathrm{~Hz})$ were increased during both movement types over effector-specific regions of motor cortices compared with typically developing individuals or duplication carriers, with no task-related performance differences between cohorts, even when corrected for their own cognitive and sensorimotor deficits. Reduced left hemispheric language specialization was observed in deletion carriers but not in duplication carriers. Neural activity over sensorimotor cortices in deletion carriers was linearly related to clinical measures of speech and motor impairment. These findings link insufficient copy number repeats at 16p11.2 to excessive neural activity (e.g., increased beta oscillations) in motor cortical networks for speech and hand motor control. These results have significant implications for understanding the neural basis of autism and related neurodevelopmental disorders.

Key words: 16p11.2; autism; beta rhythm; magnetoencephalography; manual; speech

Significance Statement

The recurrent $\sim 600 \mathrm{~kb}$ deletion at 16p11.2 (BP4-BP5) is one of the most common genetic etiologies of ASD and, more generally, of neurodevelopmental disorders. Here, we use high-resolution magnetoencephalographic imaging (MEG-I) to define with millisecond precision the underlying neurophysiological signature of motor impairments for individuals with 16p11.2 deletions. We identify significant increases in beta $(12-30 \mathrm{~Hz})$ suppression in sensorimotor cortices related to performance during speech and hand movement tasks. These findings not only provide a neurophysiological phenotype for the clinical presentation of this genetic deletion, but also guide our understanding of how genetic variation encodes for neural oscillatory dynamics.

\section{Introduction}

Sensorimotor deficits are a prevalent feature in developmental disorders, including autism spectrum disorders (ASDs; Dawson

Received Oct. 16, 2017; revised June 21, 2019; accepted June 22, 2019.

Author contributions: L.B.N.H., T.L.L., W.K.C., T.P.L.R., P.M., E.H.S., and S.S.N. designed research; L.B.N.H., T.L.L., A.M.F., P.B., N.P., T.T., and J.B. performed research; L.B.N.H., C.L.D., J.B., and S.S.N. analyzed data; L.B.N.H., E.H.S., and S.S.N. wrote the paper.

This work was supported by a Grant from the Simons Foundation to E.H.S. (SFARI award 220843), NIH Grants R01NS050915, K24DC015544, R01DC004855, R01DC010145, R21NS076171, R01DC013979, support from Hearing Research, and an NSF BCS (1262297) Grant. We thank John Spiro of the Simons Foundation for his contributions to this paper, and all of the families at the participating Simons Variation in Individuals Project (Simons VIP) sites, as and Watling, 2000; Whyatt and Craig, 2013) and its most common genetic etiology, the $16 \mathrm{p} 11.2$ (BP4-BP5) copy number variation $(\mathrm{CNV})$, which leads to one or three copies of this chromosomal region (Kumar et al., 2008; Weiss et al., 2008; Spiro and Chung, 2012; Zufferey et al., 2012). Although reductions in IQ and cognitive function are common across both duplication

well as the Simons VIP Consortium. We appreciate obtaining access to phenotypic data on SFARI Base (data pull dated December 10, 2014). Approved researchers can obtain the Simons VIP population dataset described in this study (URL of population from SFARI base) by applying at https://base.sfari.org.

The authors declare no competing financial interests.

*E.H.S. and S.S.N. contributed equally to this work. 
Table 1. Demographic data on the TD, 16p11.2 CNV DEL, and 16p11.2 CNV DUP child and adult cohorts for picture naming and linguistic categorization

\begin{tabular}{|c|c|c|c|c|c|c|c|c|c|c|c|c|}
\hline & \multicolumn{6}{|c|}{ Picture naming } & \multicolumn{6}{|c|}{ Linguistic categorization } \\
\hline & \multicolumn{3}{|l|}{ Children } & \multicolumn{3}{|l|}{ Adults } & \multicolumn{3}{|l|}{ Children } & \multicolumn{3}{|l|}{ Adults } \\
\hline & TD & DEL & DUP & TD & DEL & DUP & TD & DEL & DUP & TD & DEL & DUP \\
\hline Age & $11.8 \pm 2.6$ & $11 \pm 2.5$ & $9.8 \pm 2.5$ & $36.1 \pm 7.5$ & $38.6 \pm 7.5$ & $37.4 \pm 9.5$ & $12 \pm 2.7$ & $11.5 \pm 2.3$ & $11 \pm 2.5$ & $40.4 \pm 5.8$ & $36.9 \pm 10.3$ & $37.9 \pm 7.9$ \\
\hline$M: F$ & $14: 14$ & $14: 14$ & $10: 4$ & $6: 2$ & $6: 2$ & $12: 10$ & $12: 7$ & $12: 7$ & $3: 5$ & $6: 3$ & $6: 3$ & $6: 3$ \\
\hline L:R & $7: 21$ & $7: 21$ & $3: 11$ & $1: 7$ & 1:7 & 4:18 & $4: 15$ & $4: 15$ & $2: 6$ & $1: 8$ & 1:8 & 1:8 \\
\hline
\end{tabular}

and deletion carriers of the 16p11.2 CNV, deletion carriers exhibit pronounced coordination and speech and language deficits and to a lesser degree in reciprocal duplications (Hanson et al., 2010, 2015; Hippolyte et al., 2016; Fedorenko et al., 2016; Steinman et al., 2016). The prominence and selectivity of sensorimotor deficits in 16p11.2 deletion carriers suggest that two copies of the genes in this region are necessary for normal neural circuits driving sensorimotor control.

Previously, we have identified consistent, penetrant changes in brain anatomy and function related to $16 \mathrm{p} 11.2 \mathrm{CNV}$ cognitive and behavioral features, including increased regional brain volume (Qureshi et al., 2014), abnormal white matter microstructure (Owen et al., 2014; Berman et al., 2015, 2016; Chang et al., 2016) and delays in early auditory-evoked responses (Berman et al., 2016), providing a foundation to develop a neuroanatomical and neurophysiological "phenotype" for this genetically-defined population. The sensorimotor impairments in $16 \mathrm{p} 11.2$ deletion carriers provide a unique opportunity to link CNV to function. Here, we conducted a neuroimaging study in $16 \mathrm{p} 11.2$ probands (deletions and duplications) to examine the specificity of copy number repeats in this region on sensorimotor brain network activity. We hypothesized that deletion carriers will possess abnormal cortical neurophysiology patterns that in turn may be related to behavioral deficits.

One robust neurophysiological signature of motor behavior is the beta $(12-30 \mathrm{~Hz})$ sensorimotor cortical rhythm (Crone et al., 1998; Pfurtscheller and Lopes da Silva, 1999; McFarland et al., 2000; Pfurtscheller et al., 2003). Movement of any body structure (effector) is preceded by and is concurrent with a decrease in beta power over sensorimotor cortices as a marker of neural activity in non-invasive neuroimaging (Salmelin and Hari, 1994; Graimann et al., 2002; Müller et al., 2003; Jurkiewicz et al., 2006). Beta oscillations have been studied extensively both during hand movements (Pfurtscheller et al., 1997; Taniguchi et al., 2000; Waldert et al., 2008), and during speech preparation and production (Findlay et al., 2012; Hinkley et al., 2016). Given the impairments in speech and motor control in 16p11.2 deletion carriers, we evaluate whether the collection of genes in this region are responsible for regulating sensorimotor cortical beta oscillations. Left-lateralized beta power decreases during speech are consistent with left hemisphere language dominance. We also examine altered language lateralization in 16p11.2 deletion carriers, because atypical language lateralization has been reported in other neurodevelopmental disorders (Kleinhans et al., 2008; Knaus et al., 2010; Lindell and Hudry, 2013). Because the 16 p11.2 region is flanked by low copy number repeats, studies of individuals with 16p11.2 CNVs can act as a strong, consistent model for understanding how genetic variation impacts neurophysiology, as gen-

Correspondence should be addressed to Srikantan S. Nagarajan at sri@radiology.ucsf.edu or Elliott H. Sherr at sherre@neuropeds.ucsf.edu.

https://doi.org/10.1523/JNEUROSCI.3001-17.2019

Copyright $\odot 2019$ the authors erally the same genomic coordinates are missing, and we only included individuals with this shared deletion in our study (Zufferey et al., 2012). If the 16 p11.2 region encodes genes that regulate language laterality, then the absence of one copy of these genes would alter the hemispheric asymmetry of neurophysiological oscillations known to represent language laterality (e.g., beta rhythms). Finally, we assay whether sensorimotor beta oscillations are associated with clinical motor assays in $16 \mathrm{p} 11.2$ CNV carriers.

Here, we use high-resolution magnetoencephalographic imaging (MEGI) to define with millisecond precision the underlying neurophysiological signature of $16 \mathrm{p} 11.2$ deletion carrier motor impairments. We adapted two motor tasks (picture naming and manual button press) known to induce beta power changes and be easy enough to ensure equal and high compliance across all groups. We compare activation in deletion carriers against both matched typically developing and duplication carrier cohorts With this approach, we test three hypotheses in the 16 p11.2 deletion carriers: (1) there are atypical patterns of effector-specific sensorimotor beta oscillations in tasks matched for performance across groups, (2) atypical lateralization of language, and (3) the magnitude of aberrant sensorimotor activity is associated with the degree of clinical impairments of speech and hand movement function.

\section{Materials and Methods}

Experimental design. All participants were recruited through the Simons VIP Connect website (The Simons VIP Consortium, 2012) and gave written informed consent to participate. The recruitment arm for the Simons VIP project was explained in detail by Spiro et al., 2012. From the larger cohort recruited as part of the Simons VIP, a subset of the participants able to engage in the simplified motor tasks and able to hold still for unsedated scans were scanned in MEG and MRI at either the University of California, San Francisco (UCSF) or the Children's Hospital of Philadelphia (CHOP; Table 1). All participants were administered cognitive measures by trained child psychologists. Cognitive and language measures used for the current analysis included the non-word repetition task from the Comprehensive Test of Phonological Processing (CTOPP; Wagner et al., 1999) as a measure of basic phonological production as well as the Perdue Pegboard Test (PPB; Tiffin, 1968) as a measure of manual dexterity. From this subset of participants, 28 16p11.2 deletion carrier children (DEL), 14 16p11.2 duplication carrier children (DUP), and 28 typically developing (TD) control children matched to the proband cohorts for age, gender, and handedness that performed the picture naming (PN) task were selected (Table 1). These participants were also selected based on data quality (e.g., task compliance, lack of noisy MEG sensor data). All TD individuals were screened for $\mathrm{CNV}$ s through genetic testing and were not found to carry CNVs for 16p11.2. A similar selection process was used for $1916 \mathrm{p} 11.2$ deletion carrier children and 8 duplication carrier children (a subset of whom also underwent PN; Table 1) and 19 matched controls for the linguistic categorization task. All individuals who participated in the MEG analyses were screened for cortical and subcortical brain malformations by a neuroradiologist (P.M.) and any differences in observable malformations were not found to be statistically significant between the DUP, DEL, and TD cohorts. All participants were 
administered both the Autism Diagnostic Observation Scale (Lord et al., 2000) and the Autism Diagnostic Interview-Revised (Rutter et al., 2003) to evaluate diagnosis for ASD. Of the 28 deletion carrier participants who underwent PN in MEG, only 8 (28.6\%) met diagnostic criteria for ASD, whereas only $4 / 19$ participants who underwent linguistic categorization LC in MEG (21\%) met diagnostic criteria for ASD. Similarly, in the 14 duplication carrier children who underwent PN in MEG, only 1 (7.4\%) met diagnostic criteria for ASD, with none $(0 \%)$ of the 8 duplication carrier children who underwent LC in MEG met this diagnostic criteria. In addition to the DEL/DUP/TD children recruited for MEG scanning, a small cohort $(n=9)$ of $16 \mathrm{p} 11.2$ adult deletion carriers and larger cohort of 16p11.2 adult duplication carriers and TD controls $(n=22)$ were recruited and scanned in MEG for both the PN and LC tasks matched for age, gender, and handedness. Disparities in recruitment between deletion carriers (largely children) and duplication carriers (largely adults) are thought to be the result of a known ascertainment bias present within 16p11.2 research (Steinman et al., 2016).

MRI data acquisition. MRI data were acquired from each participant at one of the two sites (UCSF, CHOP) using identical 3.0T Siemens Trio machines. For each subject, a high-resolution anatomical MRI was acquired (MPRAGE; $1601 \mathrm{~mm}$ slices, $\mathrm{FOV}=256 \mathrm{~mm}$, TR $=2300 \mathrm{~ms}$, $\mathrm{TE}=2.98 \mathrm{~ms}$ ) to reconstruct MEG data in source space.

MEG data acquisition. MEG data were collected from each participant at one of the two sites (UCSF, CHOP), using an identical whole-head 275 axial gradiometer MEG system with third-order gradient correction (MEG International Services) at a sampling rate of $1200 \mathrm{~Hz}$. Three fiducial coils (nasion, left/right preauricular) were placed to localize the position of the head relative to the sensor array. Head localization was performed at the beginning and ending of the collection to register head position and to measure head movement during the task.

Vocal production (picture naming task). Twenty-eight children who carried deletions of 16p11.2, 28 matched controls, and 14 children who carried duplications of 16p11.2 (Table 1) participated in a picture naming task to examine motor activation before vocal production (VP). This task was designed so that it was easy enough for all participants, including deletion carriers. For the test, an image of an object is projected onto a screen (100 trials) and participants are instructed to name the pictured object into the microphone. Vocal responses were digitized on separate analog-to-digital channels (ADCs), and marked through amplitude threshold detection and verified by hand through visual inspection manually in each dataset.

Manual movement (linguistic categorization task). Nineteen children who carried deletions of 16p11.2, 19 matched controls, and 8 children who carried duplications of 16p11.2 (Table 1) participated in a picture naming task to examine motor activation during manual movements (MMs). Like the picture naming task, this test was designed to be engaging yet simple enough that performance would be comparable across both cohorts. For the test, participants are presented in the auditory domain a word and are instructed to make a judgment (either living or non-living) by pressing a button with their dominant hand (100 trials). Auditory stimulus onset (object) and manual responses were digitized on separate ADCs and marked through amplitude threshold detection and verified by hand through visual inspection manually in each dataset.

$M E G$ data analysis. All MEG datasets were preprocessed by excluding noisy MEG sensors and trials with artifacts (i.e., eye blink, EMG artifact, or other obvious sensor artifact exceeding 10pT) before source-space analysis. Trials with no responses and clear false starts (responses $\sim 300$ $\mathrm{ms}$ before stimulus presentation) were removed from the datasets. Following preprocessing, datasets were reconfigured into a response-locked format, where time point 0 was either the onset of the vocal response (VP) or the onset of the button press (MM).

MEG sensor data were reconstructed in source space using a timefrequency optimized adaptive spatial filtering technique implemented in the Neurodynamic Utility Toolbox for MEG (NUTMEG; http://nutmeg. berkeley.edu). Here, we examine induced changes in oscillatory activity during the task. First, raw sensor data are passed through several filter banks using a finite impulse response filter encompassing the beta (12-30 $\mathrm{Hz}$ ), gamma $(30-55 \mathrm{~Hz})$, and high-gamma $(65-90 \mathrm{~Hz})$ frequency bands, and partitioned into partially overlapping time windows (300 ms width for beta and low gamma, $100 \mathrm{~ms}$ width for high gamma; $50 \mathrm{~ms}$ step size) optimized for localizing spectral peaks in the MEG data (Dalal et al., 2008). Tomographic volumes of source locations (voxels, $5 \mathrm{~mm}$ lead field) are generated through computation of a covariance matrix and weights of each location relative to the signal of the MEG sensors (Dalal et al., 2008). Source power for each location was derived through a noisecorrected pseudo- $F$ statistic expressed in logarithmic units (decibels) comparing signal magnitude during an "active" experimental time window versus a baseline "control" window (Robinson and Vrba, 1999). From these volumes, a pseudo- $F$ statistic is obtained for each voxel, time window, and frequency band.

Statistical analysis. Anatomical MRIs in each subject were spatially normalized (standard MNI template, SPM8; https://www.fil.ion. ucl.ac.uk/spm/software/spm8/) with the resulting parameters being applied to each individual subject's reconstruction through Nutmeg. Group analyses to evaluate effects at the second level were performed with statistical nonparametric mapping (SnPM; Singh et al., 2003). For the MM task, beamformer reconstructions were left-right flipped for participants using their left hand to match for activations contralateral to the dominant hand. To minimize spatial frequency noise in the beamformer volumes, average and variance maps for each individual time window were calculated and smoothed using a Gaussian kernel with a width of $20 \times 20 \times 20 \mathrm{~mm}$ FWHM (Barnes et al., 2004). Statistical significance was estimated by obtaining a permuted distribution (through $2^{\mathrm{N}}$ possible combinations of negations) and estimating the significance of each pseudo- $F$ value from its position in this permuted distribution (Singh et al., 2003). Corrections for multiple comparisons were done at varying levels prioritizing conservative thresholds. Withingroup (nonparametric one-sample $t$ test) patterns of activation were corrected at a voxelwise familywise error rate (FWE) cutoff of $p<0.05$. Between-group (nonparametric two-sample unpaired $t$ test) comparisons were first evaluated using a conservative false discovery rate (FDR) threshold of $p<0.05$ at $q=10 \%$ (Benjamini and Hochberg, 1995). Failing this initial conservative FDR threshold, between-group comparisons were then corrected using a voxelwise cluster correction $(k=50$ contiguous voxels) at a threshold of $p<0.05$ (Dalal et al., 2011). Between-group comparisons for the statistical maps were adjusted by either VIQ (PN) or NVIQ (LC) as a covariate. Our rationale for VIQ/ NVIQ correction is twofold. First, significant differences in these measures between the two groups are to be expected based on prior investigations of this data in $16 \mathrm{p} 11.2 \mathrm{CNV}$ s. Second, previous reports in the behavioral literature (Hippolyte et al., 2016; D’Angelo et al., 2016) demonstrate that differences in neuropsychological testing are not directly related to IQ in larger $16 \mathrm{p} 11.2 \mathrm{CNV}$ cohorts. Correlations with neuropsychological metrics (CTOPP, PPB) were performed post hoc by first generating a ROI defined a priori by the results of the between-group analyses and then run voxelwise (partial correlation coefficient) between beta power and external variables adjusting for either VIQ (PN) or NVIQ (LC).

Laterality. To test the hypothesis that hemispheric dominance for language is altered in individuals who carry the $16 \mathrm{p} 11.2$ deletion, laterality index (LI) was derived from methods previously reported (Findlay et al., 2012; Hinkley et al., 2016; De Witte et al., 2016). Briefly, changes in beta oscillatory power were extracted from specified volumes-of-interest (VOIs) in the frontal lobe containing voxels labeled as inferior frontal gyrus, middle frontal gyrus or precentral gyrus. From this VOI, pseudo- $F$ values were selected from the $-350 \mathrm{~ms}$ to $-150 \mathrm{~ms}$ pre-response in response-locked analyses, and averaged within each VOI for each participant. LI was calculated by averaging across activation in the left and right VOI separately and contrasted using the following formula: $\mathrm{LI}=-1 \times$ $(L-R) /(|L|+|R|)$, where $L$ represents the averaged $F$ value in the left VOI and $R$ represents the averaged $F$ value in the right VOI.

\section{Results}

\section{Behavioral data}

Of the cohorts recruited to complete the vocal production (picture naming) task, significant reductions in IQ were observed in both group of probands. For child deletion carriers (see Table 1 


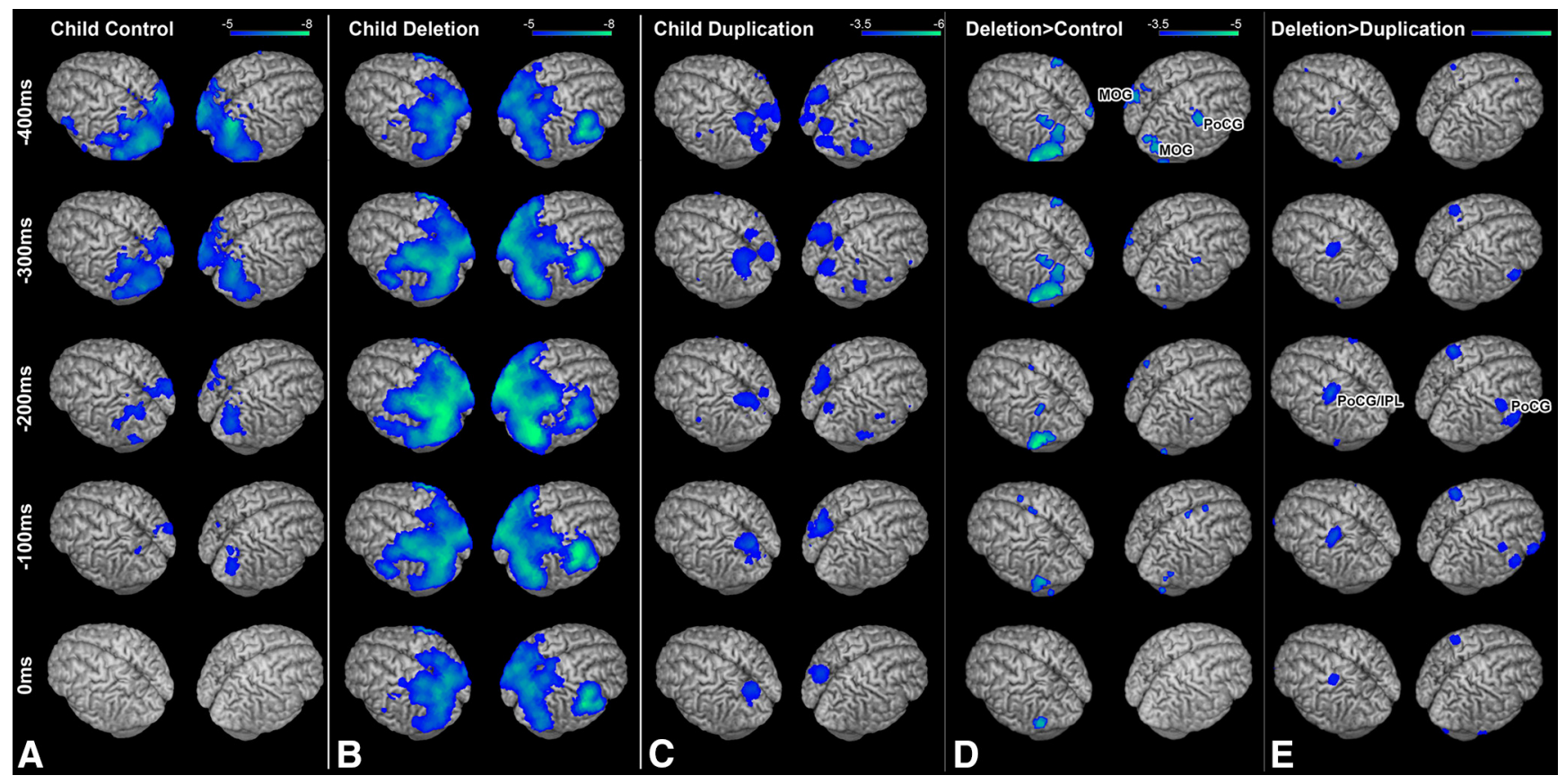

Figure 1. Response-locked $(0 \mathrm{~ms}=$ vocal production) group analyses of changes in beta $(12-30 \mathrm{~Hz})$ oscillatory power during picture naming. $A$, Within-group analysis (one-sample $t$ test) of beta power in the control cohort. Robust ( $p<0.05$ FWE) reductions in beta power are seen over occipital and parietal cortex bilaterally and over motor cortex in the left hemisphere. $\boldsymbol{B}$, Within-group analysis (one-sample $t$ test) of beta power in the 16p11.2 deletion carrier cohort. Here, activations are bilateral ( $p<0.05 \mathrm{FWE}$ ) for both occipital/parietal cortex and motor cortex. $C$, Within-group analysis (one-sample t test of beta power) in the 16p11.2 Duplication carrier cohort, exhibiting significant bilateral patterns of beta power change over the parietal cortex and motor cortex in the left hemisphere. $\boldsymbol{D}$, Comparison (unpaired nonparametric $t$ test) between Child Control and Child Deletion carrier groups. $\boldsymbol{E}$, Comparison (unpaired nonparametric $t$ test) between child deletion and child duplication carrier groups. Significant $(p<0.05 \mathrm{FDR}$ ) differences in beta power (in blue) are identifiable over the right $\mathrm{PoC} G$ when child deletions are compared between either control of duplication carrier cohorts, with increased beta power change MOG bilaterally when deletions are compared with controls and left PoCG/inferior parietal lobe (IPL) compared with duplications. All images are statistically thresholded at the multiple comparison correction levels listed above and superimposed on a MRI template brain using MRICron (http://people.cas.sc.edu/rorden/mricron/index.html).

for demographics) compared with matched controls, we observe significantly reduced full-scale IQ (FSIQ; $t_{(54)}=4.47, p=$ 0.00004 ), verbal IQ (VIQ, $t_{(54)}=5.62, p=0.7 \times 10^{-7}$ ), and performance IQ $\left(t_{(54)}=3.87\right.$, PIQ; $\left.p=0.0003\right)$. Similarly, in child duplication carriers that performed $\mathrm{PN}$, significantly reduced FSIQ, VIQ, and PIQ (FSIQ: $t_{(40)}=5.27, p=0.5 \times 10^{-6}$; VIQ: $t_{(40)}=4.36, p=0.9 \times 10^{-5}$; PIQ: $t_{(40)}=5.09, p=0.9 \times$ $10^{-6}$ ) are all readily observed compared against a normative population. These patterns were replicable in the subset of participants recruited for the manual movement (linguistic categorization) task, with lower IQ scores in both the deletion (FSIQ: $t_{(54)}=4.47, p=0.00004$; VIQ: $t_{(54)}=5.62, p=0.7 \times 10^{-7}$; PIQ: $t_{(54)}=3.87, p=0.0003$ ) and duplication (FSIQ: $t_{(25)}=3.13, p=$ 0.0036; VIQ: $t_{(25)}=3.12, p=0.0045$; PIQ: $t_{(25)}=3.47, p=$ $0.0019)$ carrier cohorts. In the neuropsychological testing data, measures of speech production (CTOPP non-word repetition task; see Materials and Methods) were also significantly reduced in deletion carriers $\left(t_{(54)}=6.76, p<0.1 \times 10^{-8}\right.$; mean $=5.42$, $\mathrm{SD}=2$ ) and in duplication carriers (mean $=7.38, \mathrm{SD}=1.66$ $\left.t_{(39)}=2.52, p=0.016\right)$ compared with the matched control cohort (mean $=8.85, \mathrm{SD}=1.7)$, indicating worse performance by the probands during that task. Similarly, neuropsychological measures of fine motor skill (PPB; see Materials and Methods) were significantly reduced in the deletion children for all measures, including performance with both hands $\left(t_{(36)}=3.196, p=\right.$ $0.0029)$, the dominant hand $\left(t_{(36)}=2.681, p=0.011\right)$, and the nondominant hand $\left(t_{(36)}=2.211, p=0.0335\right)$. For the duplication carriers, PPB scores were also significantly reduced compared with TD cohorts (both hands, $p=0.012$; dominant hand, $p=0.011$; nondominant hand, $p=0.029$ ). In contrast, during MEG scanning, no significant differences in reaction time were identifiable between deletion children, duplication children and matched controls for either the PN task (one-way ANCOVA adjusted for age and VIQ; $F=2.68, p>0.05$ ) or LC task (one-way ANCOVA adjusted for Age and NVIQ; $F=1.918, p>0.05$ ). Together, these behavioral findings suggest that both child deletions and duplications have reduced intellectual capabilities and performance impairments specific to complex motor tasks yet similar performance during the simple tasks through which we assessed their neurophysiological responses.

\section{Vocal production}

To test the hypothesis that atypical neural oscillations underlie the impairments in language production in children with 16 p11.2 deletions, we examined changes in beta power (12-30 $\mathrm{Hz}$ ) oscillations in deletion carrier children, duplication carrier children, and matched healthy controls during the picture naming task. Typically, this task induces decreases in beta $(12-30 \mathrm{~Hz})$ power primarily over language regions of the left hemisphere (Findlay et al., 2012; Hinkley et al., 2016). Here, these patterns of beta power suppression represent underlying neural activity. This pattern was observed and was highly statistically significant after stringent corrections for multiple comparisons $(p<0.05$ FWE-corrected) in the TD cohort (Fig. 1A) over regions of the occipital, temporal and parietal lobe bilaterally and the frontal lobe of the left hemisphere in the period before the subject overtly naming the object viewed on the screen (Fig. 1, 0 ms). In both sets of probands (child 16p11.2 deletion carriers and child 16p11.2 duplication carriers), a similar pattern of activation was observed with highly significant activation after stringent corrections for multiple comparisons ( $p<0.05$ FWE-corrected) over the occipital, parietal and temporal lobes, with bilateral activation in the 


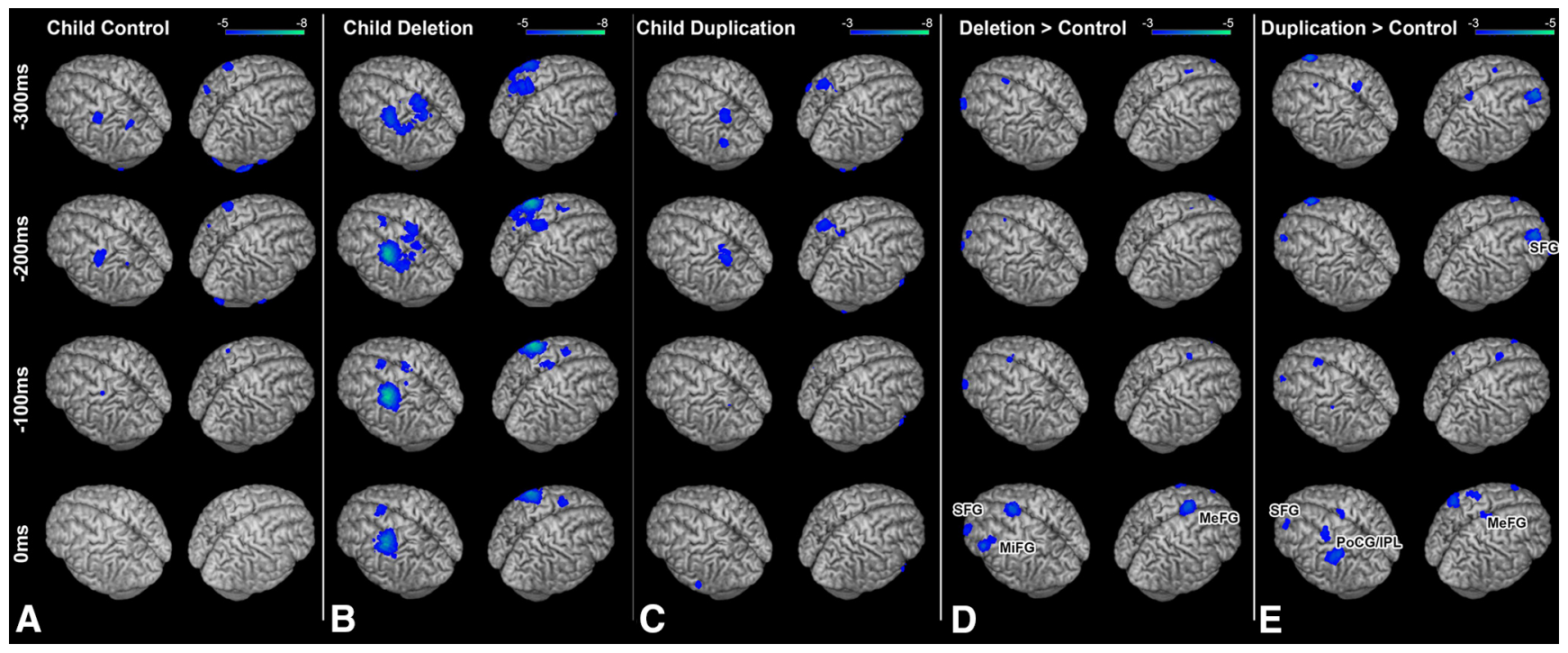

Figure 2. Response-locked ( $0 \mathrm{~ms}=$ button press) group analyses of changes in beta $(12-30 \mathrm{~Hz})$ oscillatory power during linguistic categorization. $A$, Within-group analysis (one-sample $t$ test) of beta power in the control cohort. Robust ( $p<0.05 \mathrm{FWE}$ ) reductions in beta power are seen over parietal and frontal motor cortices. $\boldsymbol{B}$, Within-group analysis (one-sample $t$ test of beta power) in the 16p11.2 deletion carrier cohort. C, Within-group analysis (one-sample $t$ test of beta power) in the 16p11.2 duplication carrier cohort. Similar patterns of activation ( $p<0.05 \mathrm{FWE}$ ) found in the control cohort are identifiable in both the deletion and duplication carrier cohorts. $D$, Comparison (unpaired nonparametric $t$ test) between child control and child deletion carrier groups. Significant differences ( $p<0.05$ cluster-corrected) in beta power (in blue) are identifiable over the left MiFG, SFG, and MeFG, with greater beta power reductions in those regions for the child deletion carrier cohort. $\boldsymbol{E}$, Comparison (unpaired nonparametric $t$ test) between child duplication and child deletion carrier groups. Significant differences ( $p<0.05$, cluster-corrected) in beta power (in blue) are identifiable over the left and right SFG and PoCG and inferior parietal lobule (IPL). Conventions as in Figure 1.

frontal lobe along the postcentral gyrus and inferior frontal gyrus (Fig. $1 B, C$ ).

We then compared activation patterns between 16p11.2 DEL and TD controls (Fig. 1D) as well as between 16p11.2 DUP and matched controls (Fig. 1E). Significantly greater neural activity was observed during this task in several key regions only in the $16 \mathrm{p} 11.2$ deletion carriers compared with either matched controls or duplication carriers. When compared against TD participants, regions over higher-order occipital cortex over the middle occipital gyrus (MOG; Fig. $1 D)$ were significantly $(p<0.05$ FDRcorrected) more active in the deletion carriers of both the left $\left(t_{(54)}=-5.374, p=1.7 \times 10^{-6}\right)$ and right $\left(t_{(54)}=-4.326, p=\right.$ $6.6^{(54)} \times 10^{-5}$ ) hemispheres. In the right hemisphere, a cluster of activation along the postcentral gyrus (PoCG; Fig. 1D) was significantly $\left(t_{(54)}=-4.498, p=3.7 \times 10^{-5}\right)$ more active in $16 \mathrm{p} 11.2$ deletion carriers than in healthy controls. These differences in activation remained statistically significant even with adjustment for VIQ (PoCG: $F=7.367, p<0.005$; right MOG: $F=10.641, p<0.005$; left MOG: $F=7.454, p<0.005)$. No regions showed reduced activation in the $16 \mathrm{p} 11.2$ deletion carriers. Significant differences between the two groups were maximal in the $450 \mathrm{~ms}$ time window before response production. When compared against child duplication carriers, similar regions of the PoCG bilaterally show significantly increased activation in the child deletion carriers $(p<0.05$ FDR-corrected; Fig. $1 E)$. Differences in activation between groups were not statistically significant for any other frequency band.

\section{Manual production}

To test the hypothesis that increased neural activity in the sensorimotor network is a general neurophysiologic feature for $16 \mathrm{p} 11.2$ deletion carriers and not specific to speech production errors, we examined activation during the response-locked (button press with the dominant hand) phase of the linguistic categorization task in both deletion carrier children and matched controls. Activation during the task (power suppression in the beta band) before the button press (0 ms) for each group (TD, DEL, and DUP carrier children) are shown in Figure 2. For both the TD (Fig. 2A) and DUP (Fig. 2C) participants, statistically significant activations $(p<0.05$ FWE-corrected) appear in the hemisphere contralateral to the hand pressing the button over posterior parietal cortex $300 \mathrm{~ms}$ before the button press and 200 $\mathrm{ms}$ before the button press over primary motor cortex. In the DEL participants (Fig. $2 B$ ), similar regions show statistically significant patterns of activity ( $p<0.05$ FWE-corrected), with additional activation over contralateral premotor cortex at $200 \mathrm{~ms}$ and over the supplementary motor area at $100 \mathrm{~ms}$ (Fig. 2B).

We then directly compared the DEL and TD cohorts during manual responses using a voxelwise nonparametric unpaired $t$ test (Fig. $2 D$ ). Statistically significant ( $p<0.05$ cluster-corrected) increases in activity were identified in the DEL group over the middle frontal gyrus $\left(\mathrm{MiFG}, t_{(36)}=-3.788, p=5.6 \times 10^{-4}\right.$ ), medial superior frontal gyrus $\left(\mathrm{MeFG} ; t_{(36)}=-3.74, p=6.4 \times\right.$ $10^{-4}$ ) and superior frontal gyrus (SFG; $t_{(36)}=-3.254, p=$ 0.0025; Fig. $2 C$ ) in the hemisphere contralateral to the hand pressing the button. No significant reductions in neural activity were identified in the DEL cohort. Furthermore, no significant differences between the two groups were identified in any of the other frequency bands. These differences (increased activation in sensorimotor regions in DEL only) remain statistically significant even when nonverbal IQ is included as a covariate in the model (SFG: $F=5.816, p=0.007$; MeFG: $F=11.461, p<0.001$; MiFG: $F=6.669, p=0.004)$. When the same comparison is made between the DEL and DUP cohorts (Fig. 2E), similar regions of the sensorimotor network show increased activation in the DEL cohort. This includes both the SFG $\left(t_{(14)}=-2.708, p=0.0177\right)$ and MeFG $\left(t_{(14)}=-2.254, p=0.0235\right)$ as well as a region of the contralateral postcentral gyrus and inferior parietal lobule (PoCG/IPL; $\left.t_{(14)}=-2.994, p=0.0076\right)$. This expands upon our finding in the vocal response dataset to verify that excessive activation in the sensorimotor network in 16p11.2 deletion carriers is not effector specific, but is seen across tasks in this cohort. 


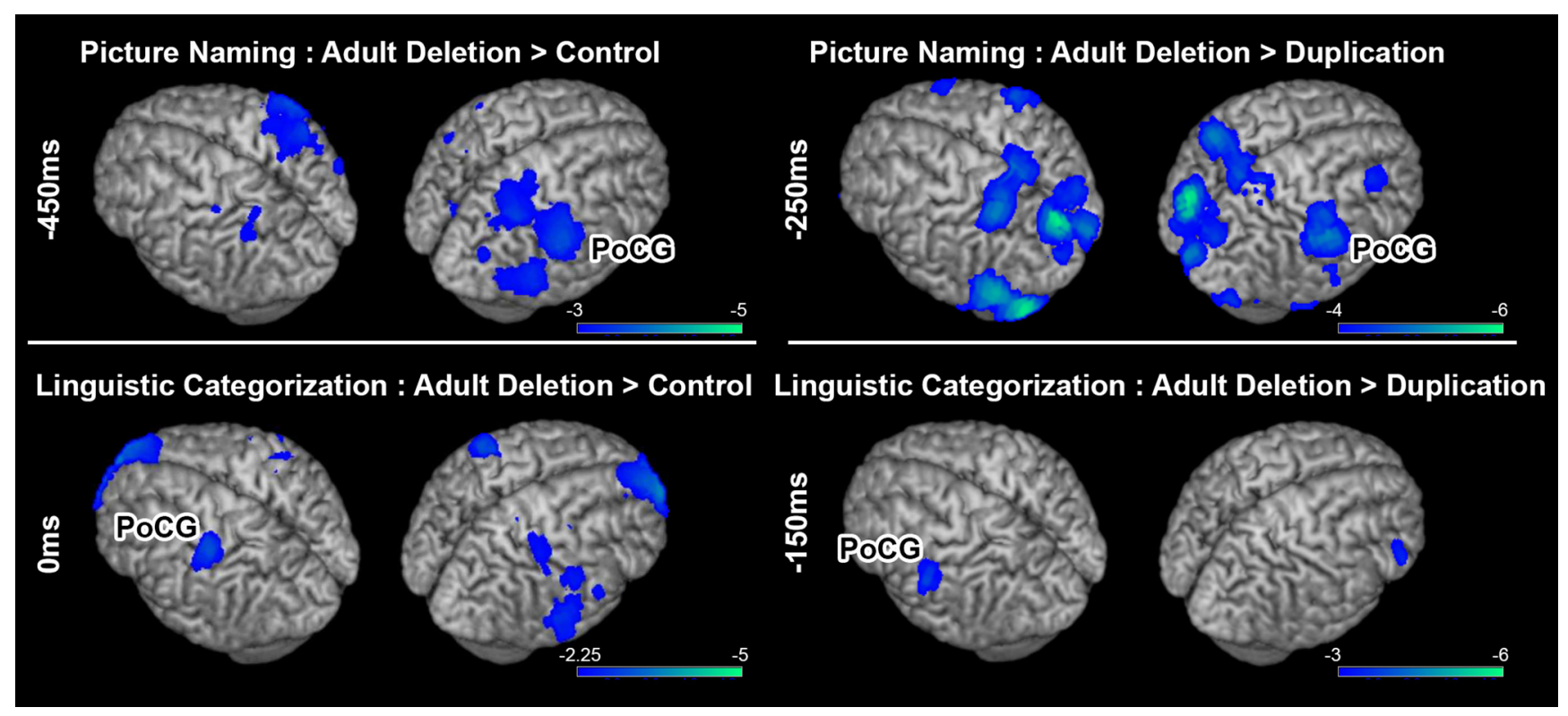

Figure 3. Results from group comparisons in adult deletion carriers against either matched controls (left) or matched adult duplications (right) for the picture naming task (top row) and linguistic categorization task (bottom row). For picture naming, increased beta power suppression was identified over the same region that showed increased activity in the child deletion carriers, over the right PoCG ( $p<0.05$ FDR-corrected). For linguistic categorization, increased activation was also identified over contralateral PoCG ( $p<0.05$ clustercorrected). Conventions as in Figure 1.

To explore whether the observed abnormal activity patterns in 16 p 11.2 children are simply the result of developmental delay or, conversely, if these patterns extend into adulthood, we examined patterns of activation (beta power changes) in the adult cohort with CNVs (either deletions or duplications) of $16 \mathrm{p} 11.2$ who performed picture naming and linguistic categorization in MEG (Table 1). One picture-naming scan in the DEL cohort was discarded due to excessive movement. These patterns of neural activity were compared with identically sized cohorts of age, gender, and handedness matched controls. Results from these group comparisons (adult DEL vs either adult TD or adult DUP) are shown in Figure 3. Similar to what was seen for picture naming in the cohort of children who carried deletions of 16p11.2, significantly increased ( $p<0.05$ FDR-corrected) neural activity was observed in adult deletion carriers compared with agematched TD controls over the right hemisphere, in the PoCG 450 ms before the response $\left(t_{(16)}=-4.83, p=1.9 \times 10^{-4}\right.$; Fig. $\left.3 A\right)$. Similarly, when adult DEL carriers are compared with adult DUP carriers, increased activation over the same region (right PoCG) is seen (Fig. $3 ; t_{(14)}=-2.113, p=0.0317$ ). In linguistic categorization, an increase in neural activity $(p<0.05$ clustercorrected) was identified before button press $(0 \mathrm{~ms})$ over the PoCG along the contralateral sensorimotor strip in the adult DEL cohort compared with either the adult TD $\left(t_{(16)}=-2.96, p=\right.$ $0.0092)$ or adult DUP $\left(t_{(16)}=-2.347, p=0.0315\right)$ cohort (Fig. $3 B)$. These findings indicate that increased activation within the sensorimotor network in deletion carriers are not a consequence of delayed development and instead represent a neurophysiological phenotype that persists into adulthood.

\section{Hemispheric dominance for language}

In addition, we used the reconstructions from the picture naming task to test the hypothesis that, as is seen in some studies of ASD, and that we demonstrated for patients with agenesis of the corpus callosum (Hinkley et al., 2016), left-hemisphere dominance for language is absent in 16p11.2 deletion carriers. Laterality esti- mates for six groups (child and adult TD, DEL, and DUP) are plotted in Figure 4. Compared with age-matched TD control participants, there is a significant $\left(t_{(56)}=-3.132, p=0.0014\right)$ reduction in laterality in the $16 \mathrm{p} 11.2$ DEL group (mean LI $=$ 0.289 ) with $17 / 28$ participants showing atypical laterality $(\mathrm{LI}<$ 0.1 ). An effect of reduced laterality is also significant when the same cohort is compared against child DUP carriers $t_{(26)}=$ $-2.987, p=0.0085$ ) indicating that this loss of laterality is specific only to those with insufficient copies of the $16 \mathrm{p} 11.2$ genes. This reduction remains statistically significant $(p<0.05)$ after VIQ and intracranial volume are included in a linear mixed effects model contrasting laterality between the two groups, indicating that this reduction in laterality is not due to differences in brain volume or IQ. Correlations between LI and age were statistically insignificant for the DEL $(r=-0.05, p>0.05)$, DUP $(r=$ $0.17, p>0.05)$, and TD $(r=0.1, p>0.05)$ cohorts. Within the DEL cohort, differences in laterality scores between DEL participants diagnosed with ASD (mean LI $=-0.0001$ ) and DEL participants without ASD diagnosis (mean $\mathrm{LI}=-0.08673$ ) were not statistically significant $(p=0.58)$ Furthermore, differences in brain malformations between the groups were not statistically significant, indicating that this shift in laterality is not confounded by clinically visible neuroanatomical differences. Patterns of laterality in the $16 \mathrm{p} 11.2$ cohort were also unrelated to hand dominance. A similar pattern is identifiable in the adult cohort who carried the deletion, with $4 / 7$ deletion carrier adults showing atypical laterality, significantly different compared with either adult TD participants $\left(t_{(16)}=-2.35, p=0.016\right)$ or adult DUP participants $t_{(14)}=-2.25, p=0.025$. This also demonstrates that this disrupted laterality is not simply a result of delayed development, as there have been reports of delayed language laterality in neurodevelopmental disorders (Finch et al., 2017). This finding is consistent with the observed increases in right hemisphere neural activity during picture naming (Fig. 1). 


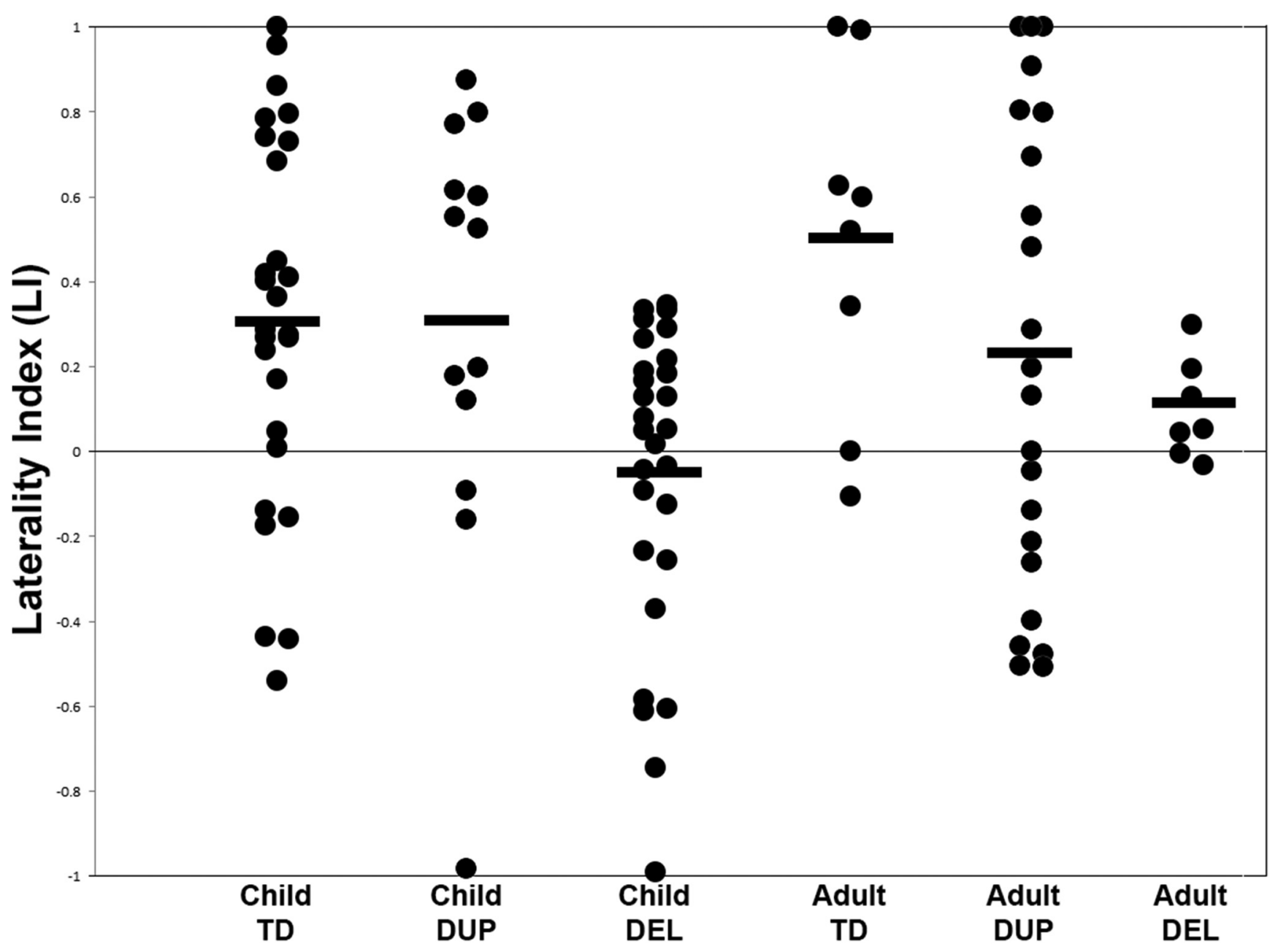

Figure 4. Scatterplot of LI derived from the picture naming task in child controls (TD), child DUP carriers, child DEL carriers, adult controls, adult DUP carriers, and adult DEL carriers. L I is estimated as a ratio between beta suppression in the left hemisphere ROI over the right hemisphere ROI, with $\mathrm{LI}>0.1$ as left-hemisphere dominant and $\mathrm{LI}<-0.1$ as right-hemisphere dominant. Significantly lower LI is identified in the child deletions compared with either the TD or DUP children $(p<0.05)$, and between the adult DEL carriers compared with either adult TD or adult DUP carriers $(p<0.05)$. Group means are plotted in horizontal bars.

\section{Correlations with neuropsychological measures}

To test the hypothesis that increased activation over the right hemisphere is related to speech production impairments in $16 \mathrm{p} 11.2$, we performed a voxelwise correlation for regions of interest defined from the group comparison for picture naming in Figure $1 C$ with a behavioral task known to be representative of the apraxic speech deficits in 16p11.2 deletions, the CTOPP nonword repetition score (Fedorenko et al., 2016). This was done separately for the three regions that were significantly different in the group analysis; the left and right MOG and the right PoCG. No significant correlations were observed within either MOG cluster for CTOPP scores (all $p>0.05$ ). However, a significant positive correlation $(r=0.554, p<0.05$ cluster-corrected) was observed between neural activity within a segment of the PoCG cluster and CTOPP scores (Fig. 5). Increased, atypically high neural activity within that region was associated with poor non-word repetition scores, suggesting a functional correlate for this heightened neural activity. Removal of outliers (beta $\mathrm{dB}$ power $>1$ ) from this analysis still produces a significant correlation $(r=$ $0.435, p<0.05$ ). No significant correlations were identified between activation in this region and CTOPP scores for either the TD cohort $(r=0.138, p=0.3121)$ or DUP cohort $(r=-0.101$, $p=0.608)$
To provide a functional substrate for excessive activation within specific regions of the sensorimotor network, we performed a voxelwise correlation separately for the contralateral MiFG, SFG, and MeFG with performance on the PPB with the dominant hand. We identified a statistically significant $(r=0.69$, $p<0.05$ cluster-corrected) correlation between neural activity in the contralateral MiFG, over the representation of the hand in premotor cortex, and performance with the dominant hand on $\mathrm{PPB}$, supporting the hypothesis that excessive neural activity leads to impaired motor performance with the dominant hand (Fig. 6). Removal of outliers (beta $\mathrm{dB}$ power $<-0.2$ ) still produces a significant correlation between PPB and MiFG power change $(r=0.448, p<$ $0.05)$. This relationship between neural activity and PPB scores are only found for the MiFG cluster. When the same correlation between PPB and neural activity is run with the SFG or MeFG cluster, no voxels were found to be correlated with PPB scores (all $p>0.05$ ). No significant correlations were identified between beta power change in these regions and PPB scores in either the TD cohort $(r=$ $-0.209, p=0.2079)$ or DUP cohort $(r=-0.2745, p=0.3035)$. This finding is consistent with our observations in picture naming and confirms that increased changes in neural activity over specific regions of the sensorimotor network are associated with more impaired motor skill. 

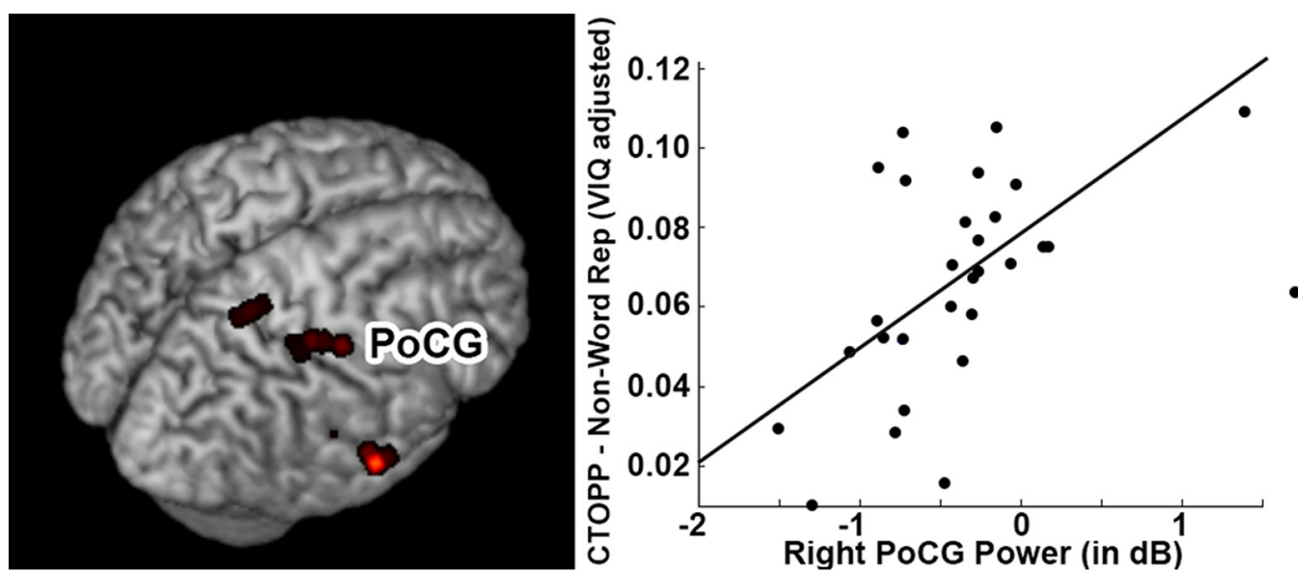

Figure 5. Association between task-induced beta power in sensorimotor cortices $(\mathrm{Po}(\mathrm{G})$ and neuropsychological variables for the picture naming task. Correlation between beta power in the right PoCG and VIQ-adjusted scores on the non-word repetition test (CTOPP). A significant ( $p<0.05$ cluster-corrected) positive correlation was identified between the two variables in child deletion carriers.
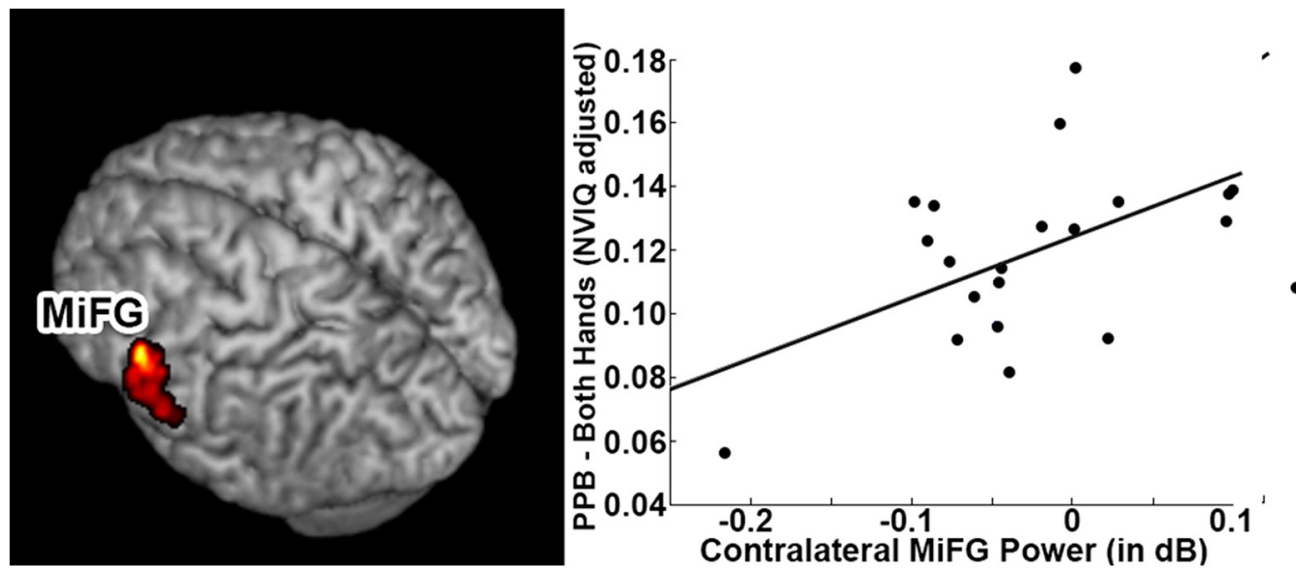

Figure 6. Association between task-induced beta power in sensorimotor cortices and neuropsychological variables for the linguistic categorization task. Correlation between beta power in the contralateral MiFG and NVIQ-adjusted scores on the PPB test. A significant positive correlation ( $p<0.05$ cluster-corrected) was identified between the two variables in child deletion carriers.

\section{Discussion}

Our study is the first of its kind to identify atypical patterns of induced sensorimotor neural activity during preparation of overt speech or hand movements specific to those with deletions, and not duplications, of 16p11.2 genes. Specifically, we found increased activation (modulation of beta oscillations) in the deletion carriers over effector-specific motor cortices during tasks designed for compliance across cohorts. Importantly, observed neural abnormalities occurred for motor tasks where there were no performance impairments in 16 p11.2 probands. We also identify reduced left hemispheric language specialization in deletion carriers, similar to observations in other neurodevelopmental disorders (Lindell and Hudry, 2013). Changes in neural activity and laterality were present in both our child and adult cohorts, suggesting that abnormal lateralization persists beyond early developmental stages in deletion carriers. Further, these changes in neural activity and laterality were only identifiable in those with deletions, and not duplications, of $16 \mathrm{p} 11.2$; suggesting a role for genes in this region in regulating sensorimotor oscillations. These findings provide an important putative functional substrate for CNV in 16p11.2; specifically, that insufficient repeats of this gene may contribute to the regulation of beta power within motor cortical networks.

We also found that increased activity in deletion carriers was associated with clinical assays of speech and motor impairment; suggesting that abnormal activity within these networks contribute to their impaired sensorimotor abilities. Prior behavioral studies in deletion carriers have found impairments in linguistic abilities (Ballif et al., 2007; Rosenfeld et al., 2010; Hanson et al., 2015) and fine motor skills (Hippolyte et al., 2016; Moreno-DeLuca et al., 2015). In speech and language domains, many of these deficits are motoric; including poor articulation, reduced speech production, and altered speech. Speech and language diagnoses are present in $71 \%$ of all individuals with this $16 \mathrm{p} 11.2$ deletion (Hanson et al., 2015) including a higher than normal prevalence of childhood apraxia of speech (Hanson et al., 2010; Hippolyte et al., 2016; Fedorenko et al., 2016). There may also be a developmental component to these speech impairments, with many studies reporting delayed expressive speech in 16p11.2 deletion carriers (Bijlsima et al., 2009; Bachmann-Gagescu et al., 2010; Shinawi et al., 2010). Findings from the current study suggest that one of the underlying mechanisms linking genes in the $16 \mathrm{p} 11.2$ region to these complex sensorimotor deficits may be aberrant regulation of neural activity over sensorimotor cortices.

Our findings favor the interpretation that alterations in neural activity found in 16p11.2 child deletions impair motor abilities, and not vice versa (e.g., altered neural activity as the result of motor impairment). Several lines of evidence in this study support this position. First, sensorimotor tasks chosen for imaging in 
our study were designed to be easy to perform for all subjects (including those with complex sensorimotor impairments), so that we can isolate patterns of neural activity unique to $16 \mathrm{p} 11.2$ deletion carriers and not confounded by performance differences. In fact, behavioral performance in our sample was comparable among 16p11.2 deletion carriers, 16p11.2 duplication carriers, and typically developing controls, supporting the notion that the effects we observe here are unaffected by difficulty during the task. Second, individuals in the 16p11.2 duplication cohort also showed deficits in motor control (reduced CTOPP and PPB scores) but did not exhibit robust increases in neural activity, similar to those seen in the deletion carrier cohort. Collectively, these findings are consistent with other observations in the literature that have shown that perturbations of sensorimotor oscillations causally impact motor performance. Transcranial stimulation of beta oscillations in motor cortex can slow and impede motor abilities in healthy individuals, suggesting that modulation of these rhythms influence motor performance ( $\mathrm{Po}-$ gosyan et al., 2009). Nevertheless, further investigations that perturb oscillations are critical to causally link excessive beta suppression in 16p11.2 CNVs and ensuing motor deficits.

Whereas sensorimotor deficits are a salient feature in $16 \mathrm{p} 11.2$ deletion carriers, they are only mildly impacted in duplications and inconsistently observed in some individuals with ASD (Dawson and Watling, 2000; Whyatt and Craig, 2013). Furthermore, although the 16p11.2 locus is one of the more common genetic variations in ASD, only one-third or less of probands meet diagnostic criteria for ASD (Hanson et al., 2015). In our study cohort, $\sim 20 \%$ of deletion carriers met diagnostic criteria for autism. Nevertheless, studies in individuals with ASD have consistently found alterations of both beta power and language laterality, consistent with the findings of the present study (Coben et al., 2008; Kleinhans et al., 2008; Puzzo et al., 2010). Overlapping reports of altered laterality in our own sample (with a significant percentage of participants with ASD) combined with reports in the literature of altered laterality in ASD suggest a common, underlying mechanism within the disorder. Furthermore, the alterations in neural activity and laterality observed in the present study in both child and adult deletion carriers strongly suggest as arising from haploinsufficiency of $16 \mathrm{p} 11.2$ copy number repeats and not general consequences of neurodevelopment. This observation is also consistent with the notion that atypical laterality is a core feature of ASD, perhaps as an inability to functionally specialize language to the left hemisphere. Atypical laterality has been suggested as a biomarker for ASD development in young children (Redcay and Courchesne, 2008; Eyler et al., 2012) as it persists into adulthood in ASD (Kleinhans et al., 2008). Furthermore, the pattern of increased right-hemisphere laterality reported here is also seen in other autism-related disorders, such as callosal agenesis (Hinkley et al., 2016), which affect brain structures (e.g., the corpus callosum) also impacted in 16p11.2 deletions (Qureshi et al., 2014). Collectively, these findings suggest that abnormal sensorimotor cortical neurophysiology and hemispheric lateralization for language function may be important components of neurodevelopmental disorders. Although a substantial proportion of the 16 p11.2 deletion participants in our own study (and others; Hanson et al., 2015) met diagnostic criteria for ASD, diagnosis status alone did not fully account for alterations in brain activity or laterality in our own study. Furthermore, altered beta activity and laterality were not influenced by factors including neuroanatomical abnormalities, brain volume, and handedness, factors known to be different in both ASD and both individuals with deletions and duplications of 16p11.2 (Hanson et al., 2015). Al- though individuals with duplications of $16 \mathrm{p} 11.2$ show minor impairments in speech and manual behavioral domains, those deficiencies cannot be accounted for by alterations in neural activity alone, speaking to perhaps an independent mechanism responsible for sensorimotor impairments in CNV duplications. Together, the relationship between CNVs of 16p11.2, neuroanatomy, sensorimotor oscillations, sensorimotor deficits, and ASD is certainly complex. Further studies in larger cohorts of children with idiopathic ASD are necessary to resolve these complicated interactions.

Here, we propose that adequate copy number repeats of 16 p11.2 are critical for coding brain oscillations for sensorimotor function. The notion supported by our data that certain brain oscillations have a genetic origin is by no means new, with many studies of monozygotic twins that have found shared components of brain oscillations (Lykken et al., 1974; Zietsch et al., 2007; van Pelt et al., 2012). Furthermore, Altamura et al. (2016) reported that, in individuals homozygous for the common polymorphism (Val/Val) of the COMT (catechol-o-methyltransferase) gene, increased beta suppression was present over the left frontal/temporal regions compared with Met/Met homozygous individuals. At a mechanistic level, therefore, the 16p11.2 CNV could include genes that are critical for the fidelity of canonical microcircuits and cortical oscillations in sensorimotor cortices (Haeusler and Maass, 2007; Bastos et al., 2012). Microcircuitry of motor cortices have been shown to generate beta rhythms (Weiler et al., 2008), and regulation of the excitatory/inhibitory balance within cortical microcircuitry could be disrupted by haploinsufficiency of genes in 16p11.2, leading to the observed aberrant oscillations in deletion carriers. This connection between genes and neurophysiology may be an important approach toward understanding complex neurodevelopmental disorders.

In this study we have shown that, when genes in 16p11.2 are insufficient, oscillations in sensorimotor cortex are dysregulated during complex human-specific behaviors (speech, hand movements). Both language and complex manual behavior are highly specialized to humans, as is expansion of cerebral cortical fields that serve such purposes (Arbib and Rizolatti, 1997; Krubitzer and Stolzenberg, 2014). If certain CNVs are genetically exclusive to our species, it is reasonable to hypothesize that their absence can directly impact more complex behaviors that make our species unique. This hypothesis is consistent with evolutionary studies of 16p11.2 that show that certain highly repetitive elements flanking this region are human specific (Nuttle et al., 2016). Further investigations combining genetics and neuroimaging will allow us to fully explore the role of specific genes that mediate neural circuitry on human-specific behaviors.

In summary, we find that the sensorimotor deficits specific to $16 \mathrm{p} 11.2$ deletion carriers are intimately related to increased neural activity over effector-specific motor cortices. It remains to be seen if this relationship between genetics, neurophysiology and behavior extend to other patterns of processing that reside within neocortical dynamics, such as the high-frequency (e.g., gamma) oscillations responsible for higher-order sensory processing (Engel and Singer, 2001). Although we focus on motor-induced beta oscillations in the present study, future investigations should incorporate how neural oscillations across the entire frequency spectrum are impacted in 16p11.2. Given the discrepancy in sample size between 16p11.2 deletion children and 16p11.2 duplication children, we acknowledge a potential limitation that studies in clinically identified participants with genetic variations can have an ascertainment bias preventing us from observing the full range of the 16p11.2 phenotype (although this potential for bi- 
ased ascertainment is not thought to be substantial; Stefansson et al., 2014). Although our findings are consistent with the autism literature, given the variable penetrance of ASD in $16 \mathrm{p} 11.2$ deletion carriers, it is certain that a more complex gene-neurophysiology relationship exists in ASD. Understanding that relationship not only requires us to define neurophysiological endophenotypes for the genetics of autism, but demands a greater appreciation for how candidate genes, neurophysiology, and behavior are linked together more generally. These types of multidisciplinary "reverse translational" approaches are important for identifying the building blocks between genetic variation and disease.

\section{References}

Altamura M, Elvevåg B, Goldberg TE, Carver FW, Weinberger DR, Coppola R (2016) The impact of Val108/158met polymorphism of catechol-Omethyltransferase on brain oscillations during working memory. Neurosci Lett 610:86-91.

Arbib M, Rizolatti G (1997) Neural expectations: a possible evolutionary path from manual skills to language. Commun Cognit 29:393-424.

Bachmann-Gagescu R, Mefford HC, Cowan C, Glew Gm, Hing AV, Wallace S, Bader PI, Hamati A, Reitnauer PJ, Smith R, Stockton DW, Muhle H, Helbig I, Eichler EE, Ballif BC, Rosenfeld J, Tsuchiya KD (2010) Recurrent 200-kb deletions of 16p11.2 that include the SH2B1 gene are associated with developmental delay and obesity. Genet Med 12:641-647.

Ballif BC, Hornor SA, Jenkins E, Madan-Khetarpal S, Surti U, Jackson KE, Asamoah A, Brock PL, Gowans GC, Conway RL, Graham JM Jr, Medne L, Zackai EH, Shaikh TH, Geoghegan J, Selzer RR, Eis PS, Bejjani BA, Shaffer LG (2007) Discovery of a previously unrecognized microdeletion syndrome of 16p11.2-p12.2. Nat Genet 39:1071-1073.

Barnes GR, Hillebrand A, Fawcett IP, Singh KD (2004) Realistic spatial sampling for MEG beamformer images. Hum Brain Mapp 23:120-127.

Bastos AM, Usrey WM, Adams RA, Mangun GR, Fries P, Friston KJ (2012) Canonical microcircuits for predictive coding. Neuron 76:695-711.

Benjamini Y, Hochberg Y (1995) Controlling the false discovery rate: a practical and powerful approach to multiple testing. J R Stat Soc B 57: 289-300.

Berman JI, Chudnovskaya D, Blaskey L, Kuschner E, Mukherjee P, Buckner R, Nagarajan S, Chung WK, Sprio JE, Sherr EH, Roberts TP (2015) Abnormal auditory and language pathways in children with $16 \mathrm{p} 11.2$ deletion. Neuroimage 9:50-57.

Berman JI, Chudnovskaya D, Blaskey L, Kuschner E, Mukherjee P, Buckner R, Nagarajan S, Chung WK, Sherr EH, Roberts TP (2016) Relationship between M100 auditory-evoked response and auditory radiation microstructure in 16p11.2 deletion and duplication carriers. AJNR 37:11781184.

Bijlsima EK, Gijsbers AC, Schuurs-Hoeijmakers JH, van Haeringen A, Fransen van de Putte DE, Anderlid BM, Lundin J, Lapunzina P, Pérez Jurado LA, Delle Chiaie B, Loeys B, Menten B, Oostra A, Verhelst H, Amor DJ, Bruno DL, van Essen AJ, Hordijk R, Sikkema-Raddatz B, Verbruggen KT, et al. (2009) Extending the phenotype of recurrent rearrangements of 16p11.2: deletions in mentally retarded patients without autism and in normal individuals. Eur J Med Genet 52:77-87.

Chang YS, Owen JP, Pojman NJ, Thieu T, Bukshpun P, Wakahiro ML, Marco EJ, Berman JI, Spiro JE, Chung WK, Buckner RL, Roberts TP, Nagarajan SS, Sherr EH, Mukherjee P (2016) Reciprocal white matter alterations due to $16 \mathrm{p} 11.2$ chromosomal deletions versus duplications. Hum Brain Mapp 37:2833-2348.

Coben R, Clarke AR, Hudspeth W, Barry RJ (2008) EEG power and coherence in autism spectrum disorder. Clin Neurophysiol 119:1002-1009.

Crone NE, Miglioretti DL, Gordon B, Sieracki JM, Wilson MT, Uematsu S, Lesser RP (1998) Functional mapping of human sensorimotor cortex with electrocorticographic spectral analysis: I. Alpha and beta eventrelated desynchronization. Brain 121:2271-2299.

Dalal SS, Guggisberg AG, Edwards E, Sekihara K, Findlay AM, Canolty RT, Berger MS, Knight RT, Barbaro NM, Kirsch HE, Nagarajan SS (2008) Five-dimensional neuroimaging: localization of the time-frequency dynamics of cortical activity. Neuroimage 40:1686-1700.

Dalal SS, Zumer JM, Guggisberg AG, Trumpis M, Wong DD, Sekihara K, Nagarajan SS (2011) MEG/EEG source reconstruction, statistical evaluation, and visualization with NUTMEG. Comput Intell Neurosci 2011: 758973.
D’Angelo D, Lebon S, Chen Q, Martin-Brevet S, Snyder LG, Hippolyte L, Hanson EMaillard AMFaucett WAMacé APain ABernier RChawner SJDavid AAndrieux JAylward EBaujat GCaldeira IConus P, Ferrari C, et al. (2016) Defining the effect of the 16p11.2 duplication on cognition, behavior and medical comorbidities. JAMA Psychiatry 73:20-30.

Dawson G, Watling R (2000) Interventions to facilitate auditory, visual and motor integration in autism: a review of the evidence. J Autism Dev Disord 30:415-421.

De Witte E, Hinkley L, Mizuiri D, Garrett C, Honma S, Kirsch H, Houde J, Berger M, Nagarajan S (2016) Language laterality assessed by magnetoencephalography (MEG) imaging using three different language tasks. Soc Neurosci Abstr 42:363:18.

Engel AK, Singer W (2001) Temporal binding and the neural correlates of sensory awareness. Trends Cogn Sci 5:16-25.

Eyler LT, Pierce K, Courchesne E (2012) A failure of left temporal cortex to specialize for language is an early emerging and fundamental property of autism. Brain 135:949-960.

Fedorenko E, Morgan A, Murray E, Cardinaux A, Mei C, Tager-Flusberg H, Fisher SE, Kanwisher N (2016) A highly penetrant form of childhood apraxia of speech due to deletion of 16p11.2. Eur J Hum Genet 24:302-306.

Finch KH, Seery AM, Talbott MR, Nelson CA, Tager-Flusberg H (2017) Lateralization of ERPs to speech and handedness in the early development of autism spectrum disorder. J Neurodev Disord 9:4.

Findlay AM, Ambrose JB, Cahn-Weiner DA, Houde JF, Honma S, Hinkley LBN, Berger MS, Nagarajan SS, Kirsch HE (2012) Dynamics of hemispheric dominance for language assessed by magnetoencephalographic imaging. Ann Neurol 71:668-686.

Graimann B, Huggins JE, Levine SP, Pfurtscheller G (2002) Visualization of significant ERD/ERS patterns in multichannel EEG and ECoG data. Clin Neurophysiol 113:43-47.

Haeusler S, Maass W (2007) A statistical analysis of information-processing properties of lamina-specific cortical microcircuit models. Cereb Cortex $17: 149-162$.

Hanson E, Nasir RH, Fong A, Lian A, Hundley R, Shen Y, Wu BL, Holm I, Miller DT (2010) Cognitive and behavioral characterization of 16p11.2 deletion syndrome. J Dev Behav Pediatr 31:649-657.

Hanson E, Bernier R, Porche K, Jackson FI, Goin-Kochel RP, Snyder LG, Snow AVWallace ASCampe KLZhang YChen QD'Angelo DMoreno-DeLuca AOrr PTBoomer KBEvans DWKanne SBerry LMiller FKOlson J, et al. (2015) The cognitive and behavioral phenotype of the 16p11.2 deletion in a clinically ascertained population. Biol Psychiatry 77:785-793.

Hinkley LB, Marco EJ, Brown EG, Bukshpun P, Gold J, Hill S, Findlay AM, Jeremy RJ, Wakahiro ML, Barkovich AJ, Mukherjee P, Sherr EH, Nagarajan SS (2016) The contribution of the corpus callosum to language lateralization. J Neurosci 36:4522-4533.

Hippolyte L, Maillard AM, Rodriguez-Herreros B, Pain A, Martin-Brevet S, Ferrari CConus PMacé AHadjikhani NMetspalu AReigo AKolk AMännik KBarker MIsidor BLe Caignec CMignot CSchneider LMottron LKeren B, et al. (2016) The number of genomic copies at the 16p11.2 locus modulates language, verbal memory and inhibition. Biol Psychiatry 80:129_ 139.

Jurkiewicz MT, Gaetz WC, Bostan AC, Cheyne D (2006) Post-movement beta rebound is generated in motor cortex: evidence from neuromagnetic recordings. Neuroimage 32:1281-1289.

Kleinhans NM, Müller RA, Cohen DN, Courchesne E (2008) Atypical functional lateralization of language in autism spectrum disorders. Brain Res 1221:115-125.

Knaus TA, Silver AM, Kennedy M, Lindgren KA, Dominick KC, Siegel J, Tager-Flusberg H (2010) Language laterality in autism spectrum disorder and typical controls: a functional, volumetric and diffusion tensor MRI study. Brain Lang 112:113-120.

Krubitzer L, Stolzenberg DS (2014) The evolutionary masquerade: genetic and epigenetic contributions to the neocortex. Curr Opin Neurobiol 24: 157-165.

Kumar RA, KaraMohamed S, Sudi J, Conrad DF, Brune C, Badner JA, Gilliam TCNowak NJCook EH JrDobyns WBChristian SL (2008) Recurrent 16p11.2 microdeletions in autism. Hum Mol Genet 17:628-638.

Lindell AK, Hudry K (2013) Atypicalities in cortical structure, handedness and functional lateralization for language in autism spectrum disorders. Neuropsychol Rev 23:257-270.

Lord C, Risi S, Lambrecht L, Cook EH Jr, Leventhal BL, DiLavore PC, Pickles 
A, Rutter M (2000) The autism diagnostic observation schedule-generic: a standard measure of social and communication deficits associated with the spectrum of autism. J Autism Dev Disord 30:205-223.

Lykken DT, Tellegen A, Thorkelson K (1974) Genetic determination of EEG frequency spectra. Biol Psychol 1:245-259.

McFarland DJ, Miner LA, Vaughan TM, Wolpaw JR (2000) Mu and beta rhythm topographies during motor imagery and actual movements. Brain Topogr 12:177-186.

Moreno-De-Luca A, Evans DW, Boomer KB, Hanson E, Bernier R, GoinKochel RP, Myers SMChallman TDMoreno-De-Luca DSlane MMHare AEChung WKSpiro JEFaucett WAMartin CLLedbetter DH (2015) The role of parental cognitive, behavioral and motor profiles in clinical variability in individuals with chromosome 16p11.2 deletions. JAMA Psychiatry $72: 119-126$.

Müller GR, Neuper C, Rupp R, Keinrath C, Gerner HJ, Pfurtscheller G (2003) Event-related beta EEG changes during wrist movements induced by functional electrical stimulation of forearm muscles in man. Neurosci Lett 340:143-147.

Nuttle X, Giannuzzi G, Duyzend MH, Schraiber JG, Narvaiza I, Sudmant PH, Penn OChiatante GMalig MHuddleston JBenner CCamponeschi FCiofi-Baffoni SStessman HAMarchetto MCDenman LHarshman LBaker CRaja APenewit K, et al. (2016) Emergence of a Homo sapiens-specific gene family and chromosome $16 \mathrm{p} 11.2 \mathrm{CNV}$ susceptibility. Nature 536:205-209

Owen JP, Chang YS, Pojman NJ, Bukshpun P, Wakahiro ML, Marco EJ, Berman JI, Spiro JE, Chung WK, Buckner RL, Roberts TP, Nagarajan SS, Sherr EH, Mukherjee P (2014) Aberrant white matter microstructure in children with 16p11.2 deletions. J Neurosci 34:6214-6223.

Pfurtscheller G, Lopes da Silva FHL (1999) Event-related EEG/MEG synchronization and desynchronization: basic principles. Clin Neurophysiol 110:1842-1857.

Pfurtscheller G, Stancák A Jr, Edlinger G (1997) On the existence of different types of central beta rhythms below $30 \mathrm{~Hz}$. Electroencephalogr Clin Neurophysiol 102:316-325.

Pfurtscheller G, Graimann B, Huggins JE, Levine SP, Schuh LA (2003) Spatiotemporal patterns of beta desynchronization and gamma synchronization in corticographic data during self-paced movement. Clin Neurophysiol 114:1226-1236.

Pogosyan A, Gaynor LD, Eusebio A, Brown P (2009) Boosting cortical activity at beta-band frequencies slows movement in humans. Curr Biol 19:1637-1641.

Puzzo I, Cooper NR, Vetter P, Russo R (2010) EEG activation differences in the pre-motor cortex and supplementary motor area between normal individuals with high and low traits of autism. Brain Res 1342:104-110.

Qureshi AY, Mueller S, Snyder AZ, Mukherjee P, Berman JI, Roberts TP, Nagarajan SS, Spiro JEChung WKSherr EHBuckner RLBuckner RL (2014) Opposing brain differences in 16p11.2 deletion and duplication carriers. J Neurosci 34:11199-11211.

Redcay E, Courchesne E (2008) Deviant functional magnetic resonance imaging patterns of brain activity to speech in 2-3-year-old children with autism spectrum disorder. Biol Psychiatry 64:589-598.

Robinson S, Vrba J (1999) Functional neuroimaging by synthetic aperture magnetometry (SAM). In: Recent advances in biomagnetism (Yoshimoto T, Kotani M, Kuriki S, Karibe H, Nakasato N, eds), pp 302-305. Sendai, Japan: Tohoku UP.

Rosenfeld JA, Coppinger J, Bejjani BA, Girirajan S, Eichler EE, Shaffer LG, Ballif BC (2010) Speech delays and behavioral problems are the predominant features in individuals with developmental delays and 16p11.2 microdeletions and microduplications. J Neurodev Disord 2:26-38.

Rutter M, Le Couteur A, Lord C (2003) Autism diagnostic interview, revised. Los Angeles: Western Psychological Services.
Salmelin R, Hari R (1994) Spatiotemporal characteristics of sensorimotor neuromagnetic rhythms related to thumb movement. Neuroscience 60:537-550

Shinawi M, Liu P, Kang SH, Shen J, Belmont JW, Scott DA, Probst FJCraigen WJGraham BHPursley AClark GLee JProud MStocco ARodriguez DLKozel BASparagana SRoeder ERMcGrew SGKurczynski TW, et al. (2010) Recurrent reciprocal 16p11.2 rearrangements associated with global developmental delay, behavioural problems, dysmorphism, epilepsy and abnormal head size. J Med Genet 47:332-341.

Singh KD, Barnes GR, Hillebrand A (2003) Group imaging of task-related changes in cortical synchronization using nonparametric permutation testing. Neuroimage 19:1589-1601.

Spiro JE, Chung WK (2012) Simons variation in individuals project (Simons VIP): a genetics-first approach to studying autism spectrum and related neurodevelopmental disorders. Neuron 73:1063-1067.

Stefansson H, Meyer-Lindenberg A, Steinberg S, Magnusdottir B, Morgen K, Arnarsdottir S, Bjornsdottir GWalters GBJonsdottir GADoyle OMTost HGrimm OKristjansdottir SSnorrason HDavidsdottir SRGudmundsson LJJonsson GFStefansdottir BHelgadottir IHaraldsson MJonsdottir B, et al. (2014) CNVs conferring risk of autism or schizophrenia affect cognition in controls. Nature 505:361-366.

Steinman KJ, Spence SJ, Ramocki MB, Proud MB, Kessler SK, Marco EJ, Green Snyder L, D’Angelo D, Chen Q, Chung WK, Sherr EH; Simons VIP Consortium (2016) 16p11.2 deletion and duplication: characterizing neurologic phenotypes in a large clinically ascertained cohort. Am J Med Genet 170:2943-2955.

Taniguchi M, Kato A, Fujita N, Hirata M, Tanaka H, Kihara T, Ninomiya H, Hirabuki N, Nakamura H, Robinson SE, Cheyne D, Yoshimine T (2000) Movement-related desynchronization of the cerebral cortex studied with spatially filtered magnetoencephalography. Neuroimage 12:298-306.

Tiffin J (1968) Purdue pegboard examiner manual. Chicago: Science Research.

van Pelt S, Boomsma DI, Fries P (2012) Mangetoencephalography in twins reveals a strong genetic determination of the peak frequency of visually induced gamma-band synchronization. J Neurosci 32:3388-3392.

Wagner RK, Torgesesn JK, Rashotte CA (1999) CTOPP: comprehensive test of phonological processing. Austin, TX: Pro-Ed.

Waldert S, Preissl H, Demandt E, Braun C, Birbaumer N, Aertsen A, Mehring C (2008) Hand movement direction decoded from MEG and EEG. J Neurosci 28:1000-1008.

Weiler N, Wood L, Yu J, Solla SA, Shepherd GM (2008) Top-down laminar organization of the excitatory network in motor cortex. Nat Neurosci 11:360-366.

Weiss LA, Shen Y, Korn JM, Arking DE, Miller DT, Fossdal R, Saemundsen E, Stefansson H, Ferreira MA, Green T, Platt OS, Ruderfer DM, Walsh CA, Altshuler D, Chakravarti A, Tanzi RE, Stefansson K, Santangelo SL, Gusella JF, Sklar P, et al. (2008) Association between microdeletion and microduplication at 16p11.2 and autism. N Engl J Med 358:667-675.

Whyatt C, Craig C (2013) Sensory-motor problems in autism. Front Integr Neurosci 7:51.

Zietsch BP, Hansen JL, Hansell NK, Geffen GM, Maritn NG, Wright MJ (2007) Common and specific genetic influences on EEG power bands delta, theta, alpha, and beta. Biol Psychol 75:154-164.

Zufferey F, Sherr EH, Beckmann ND, Hanson E, Maillard AM, Hippolyte L, Macé A, Ferrari C, Kutalik Z, Andrieux J, Aylward E, Barker M, Bernier R, Bouquillon S, Conus P, Delobel B, Faucett WA, Goin-Kochel RP, Grant E, Harewood L, et al. (2012) A $600 \mathrm{~kb}$ deletion syndrome at 16p11.2 leads to energy imbalance and neuropsychiatric disorders. J Med Genet 49: $660-668$. 